\title{
Lupus vulgaris of pinna
}

\author{
Nitin Anand · Neena Chaudhary · Chandershekhar Dravid
}

\section{Introduction}

Tuberculosis is a major health problem in developing countries. Though its incidence had decreased significantly in developed countries, its reemergence has been reported. This is due to spread of infection with human immunodeficiency virus, where tuberculosis may be the presenting illness $[1,2]$.

Tuberculosis may present itself in various ways -ranging from pulmonary tuberculosis to extra pulmonary tuberculosis. Lupus vulgaris is one of the skin manifestations of T.B. (Tuberculosis), considered classically to be the result of post primary infection in an immunocompetent patient [3]. It is supposed to arise from a tuberculous condition or a clinically inapperant tuberculous focus elsewhere in the body by haematogenous, lymphatic or contiguous spread. It is seen rarely through primary inoculation or BCG vaccination also [4]. Tuberculous involvement of mucous membrane of nose and throat is also a common source of infection. Forty percent of cases have associated tuberculous lymphadenitis or lupus of mucous membrane and 10$20 \%$ have pulmonary tuberculosis or tuberculosis of bones and joints in these patients [13].

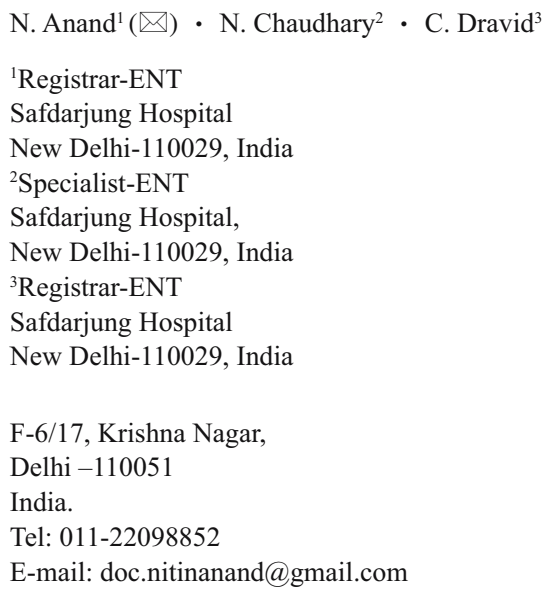

Head and neck region is involved in about $90 \%$ of patients with predilection to nose and cheek but may slowly grow to involve the adjacent areas. Cases have been seen with disseminated lupus vulgaris, where involvement of extremities and trunk are seen. The presentation of infection may vary from a macule or papule with/ without scarring and ulcerations $[5,6]$.

Despite exclusive researches being made to improve detection techniques of mycobacterium in cases of paucibacillary infection like lupus vulgaris, it still gets difficult to reach at diagnosis. Moreover, the varied morphology and rare clinical exposure to disease makes it more difficult to diagnose. In such a case it's clinical sense, which is to be relied upon instead of laboratory investigations [7, 8]. If left untreated it may lead to contractions, ulcerations and destruction of cartilaginous structures of face resulting in severe mutilation. Development of squamous cell carcinoma is one of the most worried complications seen in these patients [13].

\section{Case Report}

A 59-year-old male presented to outpatient department with complaints of skin lesion over right Pinna for past five year. It was painless and slowly progressive in nature. There was no other significant positive history. There was no positive personal history suggestive of syphilis, tuberculosis, allergy or connective tissue disorder. There was no family history of similar complaints.

On examination there was a erythematous plaque over lower half of right pinna involving both anterior and posterior surface of lobule [Fig. 1]. Purulent discharge over the lesion indicated towards the secondary bacterial infection. Diascopy revealed the typical apple-jelly appearance of nodules. Rest of the ear examination was normal. The neck examination revealed a $2 \times 2 \mathrm{~cm}$ firm, nontender, mobile right upper deep cervical lymph node.

Routine laboratory investigations were carried out. Results of tests like haemogram, with leucocyte count, ESR, 

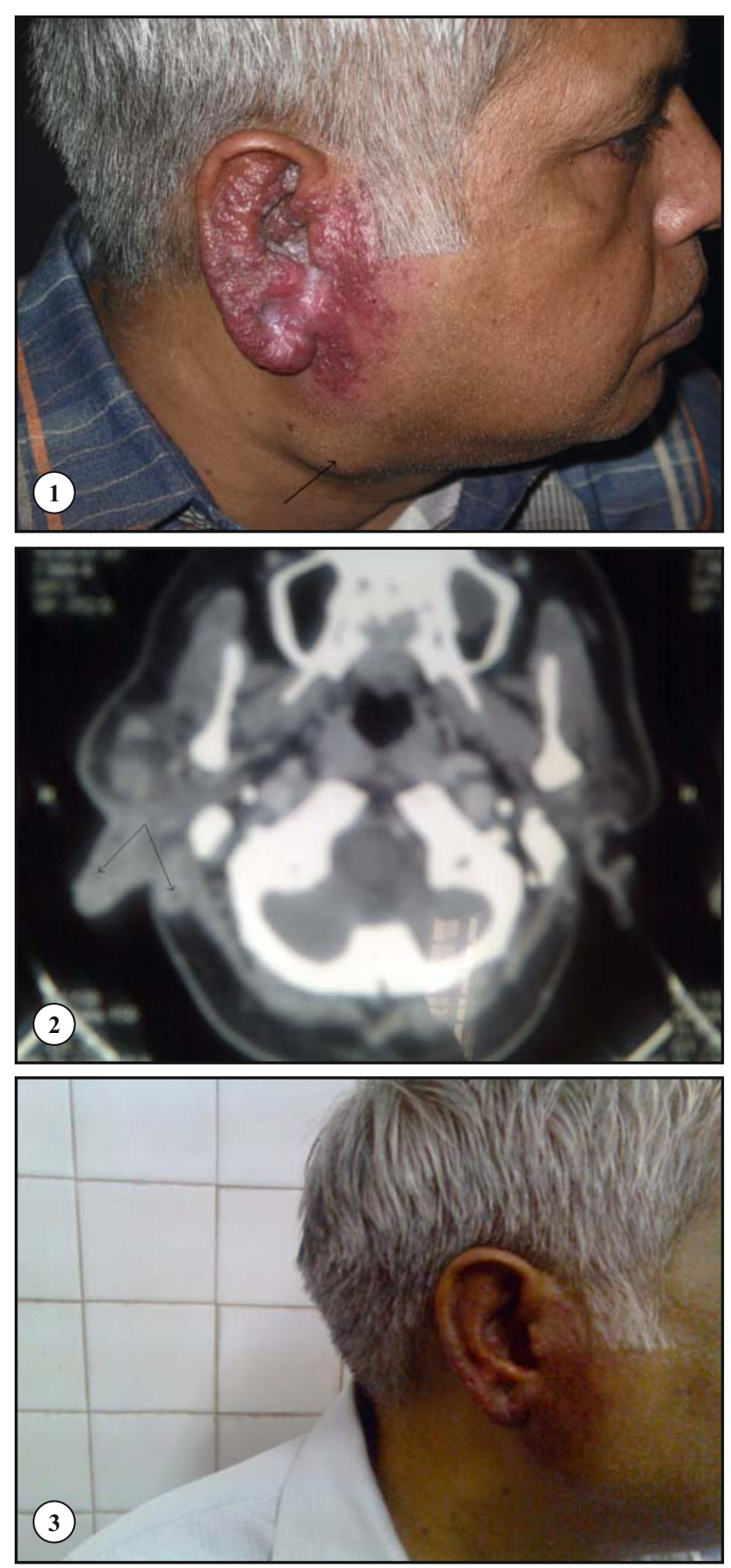

Fig. 1 Erythematous plaque over right pinna of patient and arrow pointing at the cervical lymph node

Fig. 2 CT Scan with multiple abscesses in pinna with normal middle ear Structures

Fig. 3 Post treatment hyperpigmentation of skin of pinna

kidney function test, liver function test were within normal range. Chest X-ray was normal. Mantoux test was negative. Three samples of sputum, examined for acid fast bacilli were negative. Fine needle aspiration cytology (FNAC) of the lymph node was reported as reactive Hyperplasia. Computed Tomography scan of temporal bone revealed multiple abscesses in right pinna. Middle ear appeared normal [Fig. 2]. Other specific tests like syphilis serology and antiHIV antibody titre were carried out but the results were negative. Biopsy of the lesion histologically showed noncaseating tuberculous granulomas. Few epethelioid with Langhans-type giant cells were seen but Ziehl-Neelsen staining of specimen did not demonstrate any A.F.B. Molecular test like PCR assay was unsuccessful in diagnosing tuberculous infection. Culture in Loewenstein-Jensen medium was also negative for mycobacterium.

On clinical suspicion, the patient was started on anti tubercular therapy with 4-drug regimen of isoniazid $300 \mathrm{mg}$, rifampicin $450 \mathrm{mg}$, Ethambutol $800 \mathrm{mg}$ and Pyrazinamide $1.5 \mathrm{gm}$ for first 2 months. Later the treatment was continued with 2 drugs, isoniazid and rifampicin for 7 months. After 9 months of total treatment given, patient is doing well with plaque having disappeared leaving behind post inflammatory hyperpigmentation [Fig. 3].

\section{Discussion}

Lupus Vulgaris is a chronic tuberculous infection of skin seen in previously sensitized individuals. It is seen most often after the cervical adenitis or pulmonary tuberculosis. It is supposed to reside in skin in latent form to be activated later by various stimuli [12]. Prevalence of this infection is greater in regions with cool and humid climate. It is supposed to infect patients of all ages but because of its chronic nature it is seen more in adults. Females are seen to be infected more commonly than males [6,7]. Though face is most commonly infected region, involvement of ear primarily is rare.

It presents commonly as a slowly expanding macule or plaque with an atrophic center and a raised reddish brown border. The surface of the plaque is smooth, covered with scales. Depending upon the progression of disease, ulcerations or scarring may also be seen in lesions. In hypertrophied forms it may present as tumorous growths of soft consistency [13]. On diascopy, one can see apple-jelly appearance of nodules. This is more prominently seen with small nodules at the edge of lesion [3]. For definite diagnosis of lupus Vulgaris, the demonstration of Acid Fast Bacilli (AFB) in tissues is necessary. It can be demonstrated by microscopy, culture or PCR assay. For microscopic detection of AFB, the presence of $10^{4}$ bacteria/ml of tissue is considered necessary. But in paucibacillary lesion like lupus vulgaris, bacteria are rarely demonstrated by microscopy. Moreover, microscopy cannot differentiate between typical and Atypical Mycobacterium [9]. The histopathology of the tissue shows the formation of typical tubercles with epithelioid cells, langhans giant cells and a peripheral zone of lymphocytes. Caseous necrosis is rare or may be absent 
in these cases. The culture of specimen remains unsuccessful in demonstrating bacteria in these paucibacillary lesions. The period of 6 weeks, which the culture takes, results in delay of diagnosis and treatment.

In last few years, molecular methods like PCR allows detection of mycobacterium DNA in different tissue specimens with high sensitivity $[10,11]$. It also permits rapid distinction between Typical and Atypical mycobacteria. This test has been reported to have sensitivity of around $50-72 \%$ in detection of mycobacteria [11]. But in lupus vulgaris, even this test may be negative $[7,8]$.

In our case, all laboratory investigations for detection of mycobacterium tuberculosis proved to be negative - Sputum examination, microscopy of biopsied tissue, culture for AFB and PCR Assay. Only positive finding pointing towards the diagnosis was histological picture of non caseating granulomas with few epithelioid and Langhans cells.

In contrast to sarcoidosis, where the granulomas are present in the dermis and underlying fat, here the granulomas were limited to just beneath the epidermis. Moreover there was no family history suggestive of sacoidosis.

Differential diagnosis ruled out was Leishmaniasis, which is endemic in certain areas of India. Lupoid Leishmaniasis may present itself as a tuberculous granuloma without caseous necrosis. Hisptopathological examination and Giemsa Staining may demonstrate infected histiocytes but in long standing cases, parasites may be absent from lesion. Case was reported where it had been confused with haemangioma because all definite tests for tuberculosis were negative. The excisional biopsy sent, revealed it to be lupus vulgaris [7]. Other conditions taken into consideration were sarcoidosis, discoid lupus erythematosus, leprosy, cutaneous syphilis and deep mycotic infections.

This condition has been treated with nine months regime of Autituberculous drugs like Isoniazid, Rifampicin, pyrazinamide and ethambutal. Even long term therapy with two drugs - Isoniazid and Rifampicin has been reported with success in treating this condition [5].

Various complications like joint contractures destruction of structures, disfigurement, scarring and squamous cell carcinoma have been reported in long standing cases [6] but in our case except hyperpigmentation of skin no other complication occurred.
Henceforth, we conclude here that in country like India, with a high prevalence rate of Tuberculosis, the lupus vulgaris forms an important differential diagnosis for any graulomatous infection of skin.

\section{References}

1. Huebner RE, Castro KG (1995) The changing face of tuberculosis. Annu. Rev. Med 46:47-55

2. Sunderam G, McDonald RJ, Maniatis T. et al. (1986) Tuberculosis as a manifestation of the Acquired Immunodeficiency Syndrome. JAMA 34:362- 366

3. Dan Lipsker, Edouard Grosshans (2005) What is Lupus Vulgaris in 2005? Dermatology 211(3):189-190

4. Marcussen PV (1954) Lupus Vulgaris following BCG vaccination. Br J Dermatol 66:121

5. Werschler WL, elgart ML, Williams CM (1990) Progressive asymptomatic annular facial skin lesion. Arch Dermatol; 126:1225-1230

6. Heller GL, Pavlidakey G.P., Hashimoto K et al. (1984) Lupus Vulgaris responding to double antituberculous therapy. Cutis $34: 481-483$

7. Mutsumi Okazaki, Atsushi Sakurai (1997) Lupus Vulgaris of ear lobe. Ann Plast. Sung 39(60):643-646

8. Akoglu G, Karaduman A, Boztepe G, Ozkaya O, Sahin 5, Erkin G, Kolemen F (2005) A case of lupus vulgaris successfully treated with antitubercular therapy despite negative PCR and culture. Dermatology 211:290-292

9. Lin YC, Chin HC, Hsiao CH, Jee SH, Liao YH (2002) Cutaneous Mycobacterium fortuitum infection mimicking Lupus Vulgaris. Br. J. Dermatol 147:170-173

10. Arora SK, Kumar B, Sehgal S (2000) Development of a polymerase chain reaction dot-blotting system for detecting cutaneous tuberculosis. Br. J. Dermatol 142:72-76

11. Hsiaol PF, Tzen CY, Chen HC, Su Hy (2003) Polymerase chain reaction - based detection of Mycobacterium tuberculosis in tissues showing granulomatous inflammation without demonstrable acid-fast bacilli. Int. J. Dermatol 42: 281-286

12. Ustvedt HJ, Ostensen IW (1951) The relation between tuber-culosis of the skin and primary infection. Tubercle $32: 36$

13. Klaus Wolff, Gert Tappeiner. Mycobacterial diseases: Tuberculosis and Atypical Mycobacterial Infections. In Thomas B. Fitzpatrick, Arthur Z. Elisen, Klaus Wolff, Irwin M. Freedberg, K. Frank Austen (ed), Dermatology In General Medicine 3rd edition, volume II:2158-2161 